\title{
A REVIEW ON MOTION CORRECTION METHODS IN PET/CT IMAGES FOR DETECTION OF CANCER CELLS
}

\author{
F. Nayyeri \\ Department of Computer Science \\ Faculty of Information Science and Technology \\ University Kebangsaan Malaysia
}

\begin{abstract}
Summary. Positron Emission Tomography (PET) is an important cancer imaging tool, both for diagnosing and staging, as well as offering predictive information based on response. PET is a nuclear medicine imaging technique which produces a three-dimensional image of functional processes in the body. While PET is commonly used to detect the tumors, especially in breast, colon, lung and for lymphoma, as well in the last decade it is verified as considerably more accurate than Computed Tomography (CT) in the distinction between benign and malignant lesions. PET is not only more accurate than conventional imaging for the assessment of therapy response, but also it is useful to detect some viable tumor cells after treatment. However, motion is a source of artifacts in the medical imaging and results in reducing the quantitative and qualitative accuracy of the image. In general during the procedure of PET scanning, a few types of motion can occur that should be corrected and compensated. Different body motions are classified as brain motion, cardiac motion and respiratory motion. In this study, some of the most important motion correction and compensation methods using PET imaging system are compared.
\end{abstract}

Key words: positron emission tomography, computed tomography, motion correction, motion compensation

\section{INTRODUCTION}

Co ensure optimal treatment, an accurate and reliable staging modality is essential (Takeuchi, Khiewvan et al. 2014). The conventional imaging techniques of Magnetic Resonance Imaging (MRI) and Computed Tomography (CT) with high spatial and contrast resolution are commonly used but they still have expass some difficulty to localize, visualize, and evaluate abnormal re- 
gions. Convincing evidences from several sources propose that although CT screening is the best method to detect cancer cells in its earliest stage, if a lymph node is not enlarged or there is a spot in the tissue with the same density as the regular tissue, or there is other spot like a benign cyst, CT scans have limitations in detecting cancer. Both methods are potent for size and shape defining, but they can not estimate if something is metabolically active or not (Saha 2010). However, PET, as a functional imaging method, assesses the characterization and measurement of the biologic processes. PET images generally lack anatomic context and have lower spatial resolution than $\mathrm{CT}$ and $\mathrm{MRI}$, but provide quantitative information about diseases and structures (Bagci, Udupa et al. 2013).

PET scan is a potent method in determining whether some lesion is metabolically active or not, but it generally can not determine precisely the size of the lesion. Recently, combining unique strengths of functional and anatomical imaging modalities, PET/CT has received much attention. PET/CT is the combination of PET and CT imaging techniques within a single machine. The individual PET and CT scans are taken concurrently while the patient remains in place, and can be presented separately or as a single, overlapping, "fused" image (Takeuchi, Khiewvan et al. 2014).

In this context, patient's movement becomes a major factor that degrades the image quality. CT images are acquired within a few seconds, whereas PET images are obtained over several minutes for each axial field of view. Thus it is important to take movements into account and respectively correct PET data. As a result, it will considerably benefit PET-based diagnosis and will make research in that field more reliable and accurate.

\section{GLOBAL CANCER BURDEN IN 2012}

The International Agency for Research on Cancer (IARC), which is specialized cancer agency of the World Health Organization, releases the latest data on cancer incidence, mortality, and prevalence worldwide. According to GLOBOCAN Project 2012 of the IARC, an estimated 14.1 million new cancer cases and 8.2 million cancer-related deaths occurred in 2012, compared to 12.7 million and 7.6 million, respectively, in 2008. Prevalence estimated for 2012 showed that there were 32.6 million people (over the age of 15 years) alive who had had a cancer diagnosed in the previous five years (Ferlay, Soerjomataram et al. 2014). Table 1 shows the most common cancer types.

The most commonly diagnosed cancers worldwide were those of the lung (1.8 million, $13.0 \%$ of the total), breast (1.7 million, $11.9 \%$ ), and colorectum (1.4 million, $9.7 \%)$. The most common causes of cancer death were cancers of the lung (1.6 million, $19.4 \%$ of the total), liver (0.8 million, $9.1 \%$ ), and stomach (0.7 million, $8.8 \%$ ) (Ferlay, Soerjomataram et al. 2014). 
Table 1. The most common cancers in both sexes (GLOBOCAN 2012)

\begin{tabular}{|c|c|c|c|c|c|}
\hline \multicolumn{6}{|c|}{ common LOBOCAN } \\
\hline \multicolumn{3}{|c|}{ Male } & \multicolumn{3}{|c|}{ Female } \\
\hline \multirow{2}{*}{ Cancer } & \multicolumn{2}{|c|}{$\begin{array}{c}\text { ASR }^{*} \\
\text { (per 100,000) }\end{array}$} & \multirow{2}{*}{ Cancer } & \multicolumn{2}{|c|}{$\begin{array}{c}\text { ASR } \\
\text { (per 100,000) }\end{array}$} \\
\hline & Incidence & Mortality & & Incidence & Mortality \\
\hline Lung & 34.9 & 30.9 & Breast & 47.9 & 14.9 \\
\hline Prostate & 31.2 & 8.6 & Colon & 17.6 & 9.2 \\
\hline Colon & 21 & 10.5 & Lung & 16.7 & 14 \\
\hline Stomach & 17.7 & 13.2 & Cervix uteri & 15.1 & 7.6 \\
\hline Liver & 15.6 & 14.6 & Stomach & 9.2 & 7.3 \\
\hline Bladder & 9.3 & 3.5 & Corpus uteri & 9.1 & 2.2 \\
\hline Oesophagus & 9.1 & 7.9 & Ovary & 6.8 & 4.3 \\
\hline Non-Hodgkin lymphoma & 6.1 & 3.2 & Thyroid & 6.6 & 0.8 \\
\hline Kidney & 6 & 2.6 & Liver & 6.5 & 6.4 \\
\hline Lip, oral cavity & 5.6 & 2.8 & Non-Hodgkin lymphoma & 4.8 & 2.4 \\
\hline
\end{tabular}

\section{PET/CT IN DIAGNOSTIC PROCESS}

Conventional imaging modalities such as $\mathrm{CT}$ or MRI, which use only dimensional criteria to detect nodal involvement, have poor discriminatory power in differentiating benign from malignant nodal disease (sensitivity range between $60 \%$ and $83 \%$ and specificity range between $77 \%$ and $82 \%$ ) (Ambrosini, Nicolini et al. 2012). In the past decades, CT has been employed as the gold standard imaging modality for cancer cell staging. In fact, CT can accurately determine tumor size, mediastinal and vascular invasion and can also suggest lymph node involvement, when the nodal axial diameter is greater than $1 \mathrm{~cm}$. In the following figure a mass in the left lung is shown on the CT image (Figure 1a), while the combined PET/CT (Figure 1b) image reveals the metabolic activity of that mass, as well as its precise location in the lung. The fused image can help to diagnose and stage the disease, and to tailor the treatment plan.

(a) CT image

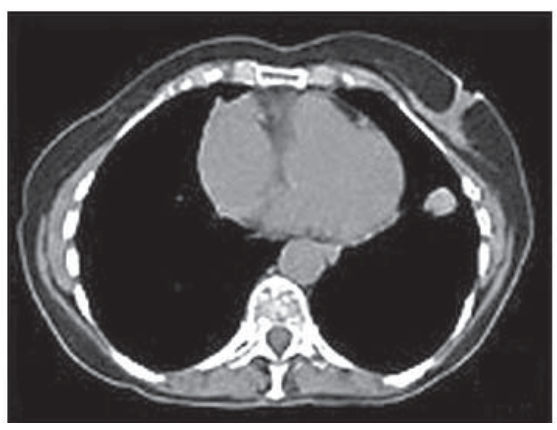

(b) PET/CT image

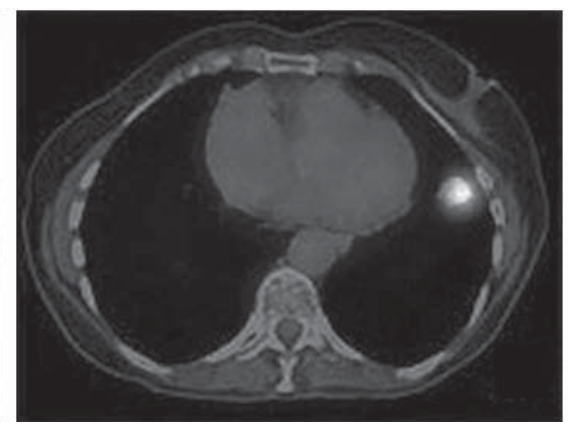

Fig. 1. A mass in the left lung shown on (a) a CT image and on (b) a combined PET/CT image (http://www.californiaheartandlungsurgery.com) 
PET/CT was reported to have a higher diagnostic accuracy than either PET or CT alone. In addition, for the assessment of tumor, the integrated PET/CT imaging has increased the accuracy of tumor detection compared to PET alone. PET/CT may provide valuable information for the assessment of nodal stations that may be missed by conventional imaging. Nevertheless, a recent cost-effectiveness analysis showed that PET/CT can be recommended from an economic point of view (Ambrosini, Nicolini et al. 2012).

\section{PET/CT IN RADIATION THERAPY (RT)}

Recent studies show that despite improvements in survival rates for many tumors, the 5-year overall survival for lung cancer remains relatively poor (around 10\%) (Saha 2010, Bagci, Udupa et al. 2013) mainly because lung cancer is often well advanced at the time of diagnosis and treatment options are limited (Takeuchi, Khiewvan et al. 2014). Although surgery is the therapy of choice in early-stage lung cancer, RT plays a major role in patients who are either medically or technically inoperable and who are not candidates for surgery (Lee, Kupelian et al. 2012).

Accurate staging of Non-Small Cell Lung Cancer (NSCLC) is essential for appropriate therapy selection. Although the definition of volumes on PET images alone might be more problematic due to the poorer resolution and higher noise levels, when combined with structural imaging, such as CT, PET provides the best available information on tumor extent.

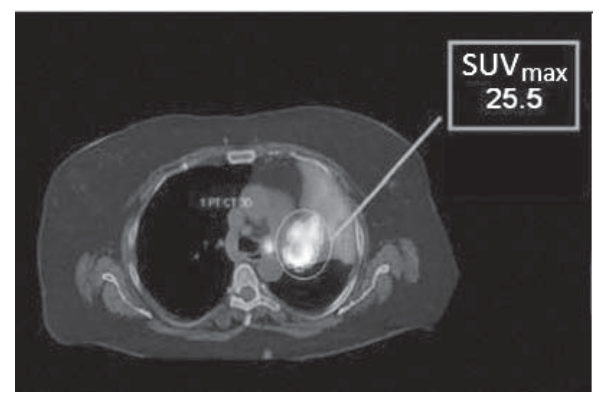

Pre-therapy

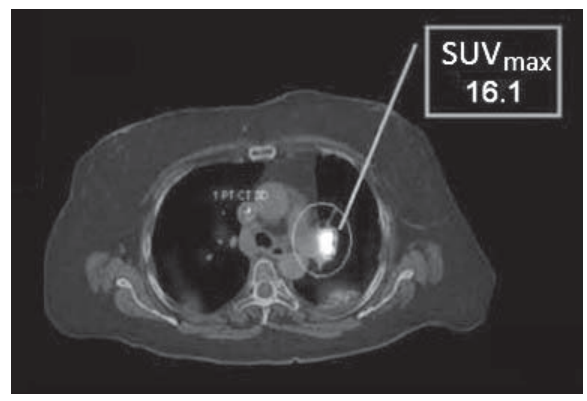

Post--therapy

Fig. 2. Post-radiation therapy with $18 \mathrm{~F}$-fluoro-2-deoxyglucose positron emission tomography integrated with computed tomography (18F-FDG PET/CT) shows significant decrease in tumor volume.

Data courtesy of M. D. Anderson, Cancer Center, Orlando, FL, USA (Lee, Kupelian et al. 2012)

In the assessment of cancer, and more recently in infection diseases diagnosing, fluorodeoxyglucose (FDG) is the most commonly used PET radiotracer that enters tumor cells provided by their increased glucose metabolism. The most metabolically active tissues have the greatest need for sugar from the bloodstream which is measured objectively as Standard Uptake Value (SUV). This is a number where higher number means a higher metabolic rate which can be due to abnormal body activities. In other 
words, increased FDG uptake in metastatic nodes, which may not be significantly enlarged by CT criteria, enhances the sensitivity of PET/CT. In fact PET/CT should be used for RT planning in NSCLC management because it displays tumor extent more accurately than CT alone. The National Comprehensive Cancer Network guideline on NSCLC states that PET/CT should be performed preferably 4 weeks before treatment (Takeuchi, Khiewvan et al. 2014). However, sequential PET/CT imaging during RT has been shown to be beneficial for accurate evaluation of therapy response. Figure 2 shows FDG PET/CT before and after RT (Lee, Kupelian et al. 2012).

\section{TYPE OF MOTION}

In general during the procedure of PET scanning, a few types of motion can occur. These different body motions are categorized as brain motion, cardiac motion and respiratory motion. Each type needs its own specific approach to correct the obtained images.

\section{Brain PET Imaging}

Unlike cardiac- and respiratory-related motions, patient movements in brain imaging are assumed to be of rigid nature. Because a typical PET brain imaging session can last hours, it is not reasonable to expect a patient to remain motionless during the whole time (Rahmim, Rousset et al. 2007). A number of head restraints are nowadays common, such as thermoplastic masks or neoprene caps that lower the amount of motion but do not eliminate it (Rahmim, Rousset et al. 2007). Even with head restraints, typical translations in the range of 5 to $20 \mathrm{~mm}$ and rotations of 1 to 4 degrees are observed, depending on the type of mask and the scan duration.

\section{Motion Due To The Cardiac Cycle}

Although a spatial resolution of less than $5 \mathrm{~mm}$ is possible with current generation PET scanners, the base of the heart moves 9 to $14 \mathrm{~mm}$ toward the apex (Rahmim, Rousset et al. 2007). Compared to the primary resolution of today's scanners, cardiac motion can therefore result in significantly blurred images. The most common approach to correct the cardiac cycle motions is gating the data into frames, where each frame represents a particular cardiac phase. Typically, the cardiac cycle is divided into 50- to 100-millisecond time frames, and an acquisition ranging from 5 to 60 minutes is usually acquired. Most commonly, the obtained cardiac-gated datasets (i.e. cardiac frames) are independently reconstructed. This approach is successful in removing nearly the cardiac-motion blurring of the images. However, it usually produces images that are much noisier than a reconstruction of the ungated data due to less statistics in each gated dataset in comparison with the entire dataset (Rahmim, Rousset et al. 2007). 


\section{Motion Caused By The Respiratory Cycle}

Respiratory motion is a source of artifacts in thoracic and abdominal organs resulting in a decrease in the quantitative and qualitative accuracy of the image. It has been shown that during a quiet breathing in the spine position the diaphragm can move by as much as $20 \mathrm{~mm}$ and thus the liver can move by an average of $11 \mathrm{~mm}$. It is also well known that breathing induces rotational and/or translational movements in thoracic and abdominal organs to varying extents. For example, upper areas of the lungs are less subject to motion compared with the lower parts (Pépin, Daouk et al. 2014). Therefore, for cancers located in the thorax or abdomen, the organ motion caused by respiration remains a limiting factor in diagnostic imaging. The simplest approach to respiratory motion issue is the "breath-holding", but this limits acquisition time to typically less than $30 \mathrm{~s}$, which is inadequate for PET imaging. Another technique, called respiratory gating, is based on gated acquisition and synchronization between the respiratory signal and PET acquisition. Respiratory gating involves only acquired imaging data during a limited window (e.g. end-expiration), based on a simple respiratory signal. However, this significantly increases acquisition time.

\section{MOTION TRACKING}

An alternative solution for the motion correction is a motion tracking. In this technique, the respiratory signal is usually produced by an external sensor that tracks a physiological characteristic related to the organ's movement. As it can be difficult to image the motion of interest directly during the procedure, markers are often implanted into the region of interest and are tracked using an imaging device such as a camera (McClelland, Hawkes et al. 2013). In this case the implantation can be invasive and motion information is only available at the marker(s) and not for the whole region of interest. Table 2 shows a summary of different types of motion correction techniques and also shows the advantages and disadvantages of each technique.

Table 2. Summary of different motion correction methods

\begin{tabular}{|l|l|l|l|}
\hline Year/Reference & Motion Area & Motion Correction Method & Results \\
\hline Li, Xie et al. 2010 & Brain motion & $\begin{array}{l}\text { Developing the 3D volumetric image } \\
\text { registration (3DVIR) with sub-mm } \\
\text { accuracy to detect and correct subtle } \\
\text { misalignment in c0-registered PET/ } \\
\text { CT head images, using infrared motion } \\
\text { tracking camera and four different head } \\
\text { holding devices to reduce motion }\end{array}$ & $\begin{array}{l}\text { Study on 53 patients found } \\
\text { misalignments in more than 80\% of } \\
\text { image sets. }\end{array}$ \\
\hline $\begin{array}{l}\text { Fayad, Pan et al. } \\
2010\end{array}$ & Respiratory motion & $\begin{array}{l}\text { Reconstructing PET frames using the } \\
\text { one-pass list mode EM (OPL-EM) } \\
\text { algorithm and a realistic non-regular } \\
\text { patient-respiratory signal phantom. }\end{array}$ & $\begin{array}{l}\text { Study on 2 nurbs-based cardiac- } \\
\text { torso (NCAT) phantoms and on } \\
\text { clinical data for 6 patients showed } \\
\text { improvement in the accuracy of the } \\
\text { PET respiratory corrected images. }\end{array}$ \\
\hline
\end{tabular}


Continned from the tabl. 2

\begin{tabular}{|c|c|c|c|}
\hline $\begin{array}{l}\text { Chang, Chang et } \\
\text { al. } 2010\end{array}$ & Respiratory motion & $\begin{array}{l}\text { Proposing a joint motion and partial } \\
\text { volume effects (PVE) correction approach }\end{array}$ & $\begin{array}{l}\text { Study on } 2 \text { phantoms and } 8 \\
\text { patients. found improvement in the } \\
\text { accuracy of PET quantification by } \\
\text { simultaneously compensating for the } \\
\text { respiratory motion artifacts. }\end{array}$ \\
\hline \begin{tabular}{|l|} 
Marache-Francisco, \\
Lamare et al. 2010)
\end{tabular} & Respiratory motion & $\begin{array}{l}\text { Evaluating two types of motion correction } \\
\text { techniques: averaging the co-registered } \\
\text { gated reconstructed PET images and } \\
\text { integrating the motion fields during the } \\
\text { iterative reconstruction process. }\end{array}$ & $\begin{array}{l}\text { Study on } 7 \text { data series found clearly } \\
\text { visible tumor on corrected image } \\
\text { compared to hardly seen and not } \\
\text { distinguishable tumor from the noise } \\
\text { on the uncorrected one. }\end{array}$ \\
\hline $\begin{array}{l}\text { Ambwani, Karl et } \\
\text { al. } 2011\end{array}$ & Cardiac motion & $\begin{array}{l}\text { Cardiac Shape Tracking with Adjustment } \\
\text { for Respiration (CSTAR) using CT images } \\
\text { for cardiac shape tracking through the } \\
\text { estimation of cardiac motion }\end{array}$ & $\begin{array}{l}\text { Study on simulated cardiac PET/ } \\
\text { CT data corresponding to the XCAT } \\
\text { phantom found qualitative and } \\
\text { quantitative improvement compared } \\
\text { with conventional PET reconstruction. }\end{array}$ \\
\hline $\begin{array}{l}\text { McQuaid, Lambrou } \\
\text { et al. } 2011\end{array}$ & Cardiac motion & $\begin{array}{l}\text { Proposing a statistical shape model } \\
\text { to describe the diaphragm shape and } \\
\text { motion (diaphragm matching between } \\
\text { PET and CT) }\end{array}$ & $\begin{array}{l}\text { Study on } 2 \text { patients revealed } \\
\text { quantitative improvements in the } \\
\text { PET images once they had been } \\
\text { corrected for attenuation and motion. }\end{array}$ \\
\hline $\begin{array}{l}\text { Keller, Sibomana et } \\
\text { al. } 2012\end{array}$ & Brain motion & $\begin{array}{l}\text { Proposing of } 3 \text { objective motion } \\
\text { correction methods with corresponding } \\
\text { results on human FDG brain scans, } \\
\text { using Polaris Vicra (Northern Digital Inc.) } \\
\text { and optical motion tracking with markers } \\
\text { attached to the head }\end{array}$ & $\begin{array}{l}\text { Study on } 17 \text { patients Pros: effective } \\
\text { in motion larger than } 2 \mathrm{~mm} \text { Cons: } \\
\text { The fixation method of the markers is } \\
\text { the main source of errors }\end{array}$ \\
\hline $\begin{array}{l}\text { Anishchenko, Hui et } \\
\text { al. } 2012\end{array}$ & Brain motion & $\begin{array}{l}\text { Proposing a head pose estimation } \\
\text { system for motion correction, using } \\
\text { external motion tracker to detect the head } \\
\text { pose with a photo camera and two web } \\
\text { cameras }\end{array}$ & $\begin{array}{l}\text { Study on } 12 \text { patients Cons: head } \\
\text { rotation should happen only in one } \\
\text { plane and relative to one axis (in } \\
\text { real conditions, head movement can } \\
\text { happen in any direction), this method } \\
\text { cannot detect head movements less } \\
\text { than the tracker precision. In this } \\
\text { situations new additional blurriness } \\
\text { will be introduced }\end{array}$ \\
\hline $\begin{array}{l}\text { Noonan, Howard et } \\
\text { al. } 2012\end{array}$ & Respiratory motion & $\begin{array}{l}\text { Developing a low cost, high accuracy } \\
\text { solution for tracking respiratory motion by } \\
\text { producing a respiratory signal phantom to } \\
\text { enable gating of PET list mode data }\end{array}$ & $\begin{array}{l}\text { Study on a National Electrical } \\
\text { manufacturers Association (NEMA) } \\
\text { image phantom data improving } \\
\text { motion correction of whole body } \\
\text { imaging by PET and camera-based } \\
\text { coincidence image techniques }\end{array}$ \\
\hline
\end{tabular}

\section{RESPIRATORY MOTION COMPENSATION IN PET/CT IMAGING}

The respiratory motion in thoracic PET images may lead to misidentification of the lesion or overestimation of its size. To solve the problem of motion compensation of PET data, integrated PET/CT scan system is performed where CT scan is acquired prior to PET acquisition. If the CT scan is performed during the normal breathing of the patient, the artifacts produced in the CT image are further transmitted into the PET image due to attenuation correction using the corrupted 
CT image. For these reasons breath-holding is recommended technique in CT acquisition. As the CT scan is completed in a few seconds, breath-holding is a possible approach. But PET data acquisition contains the whole moving process and when use a breath-hold CT scan is only used, a part of the PET data can be corrected for attenuation (Bai and Brady 2011). Due to motion, especially for lesions near the boundary between lung and liver, the intensity of a lesion is reduced, its size is overestimated and it is seriously mislocalized. These effects lead to inaccurate lung cancer diagnosing and to a staging, which depend on the intensity, the extent, and the location of a lesion. For all mentioned reasons, to yield accurate and clear images and to avoid misled diagnosis, respiratory motion in PET data must be corrected.

Therefore, to improve the level of confidence in PET scan images an improvement in respiratory motion artifacts is needed. One method to compensate these motion artifacts is the respiratory gating. In this method PET image data are acquired into discrete bins within each breathing cycle where the first bin is triggered at the user preset position in the breathing cycle. To minimize the lesion motion, the number of bins within the patient's respiratory cycle is optimized.

Nehmeh et al. in 2002 (Nehmeh, Erdi et al. 2002) developed a gating technique to account for respiratory motion which decreased the accuracy in PET imaging of lung cancer. Respiratory gating reduced the activity smearing and improved the accuracy in identifying the tumor. In this article clinical data for five patients with lung cancer showed the potential benefits of respiratory gating PET imaging.

In the study of Bettinardi et al. (Bettinardi, Picchio et al. 2010) the purpose is to describe the degradation effects produced by respiratory organ and lesion motion on PET/CT images and to define the role of respiratory gated 4D-PET/CT techniques to compensate for such effects.

He et al. (He, O'Keefe et al. 2010) proposed a new method for gating respiratory motion in a 3D PET scanner system. This non-invasive method needs no additional hardware device to utilize the geometric sensitivity of PET imaging. However, being insensitive to non-axial motion is the drawback of this method. Since the organ motion is in the " $z$ " direction within the respiratory cycle, the proposed method by simulating PET acquisition and respiratory motion is able to improve the image degradation caused by the respiratory motion.

In 2011, a method for motion and attenuation correction is proposed in respiratory gated PET images. According to experimental results this method is able to correct effectively the motion and improve the PET image quality. In addition, this method provides improved diagnostic information without increasing the acquisition duration or radiation burden. However, the major drawback of this method is using a time-varying system matrix for handling the respiratory motion. This strategy performs motion correction by a very large matrix. The dimensions of this matrix are 
determined by the number of voxels and the number of projection bins, thus calculating such a matrix carries a major challenge for computational resources (Bai and Brady 2011).

As mentioned earlier, most of the artifact corrections are based on synchronization between the respiratory signal and PET acquisition. The respiratory signal is usually produced by an external sensor that is responsible for tracking the physiological characteristic related to breathing (Pépin, Daouk et al. 2014). Respiratory gating is a technique for reconstructed PET images that excludes the motion by using time or amplitude binning. Although this technique is normally applied in clinical images, it is not able to completely correct the breathing motion because each gate can mix several tissue positions. The most important possibility to solve this problem is PET acquisition duration. The first solution is increasing the time by either selecting PET events from gated acquisitions or performing several PET acquisitions. Therefore, enough counting statistics are to be obtained in the different gates after binning. The second solution is not to increase the acquisition duration, which suggests taking into account all counting statistics and integrating motion information before, during or after the reconstruction process. In the Table 3 the literature about the respiratory motion is summarized.

Table 3. Summary of different motion correction methods

\begin{tabular}{|l|l|}
\hline Reference /Year & Summary \\
\hline Nehmeh, Erdi et al. 2002 & $\begin{array}{l}\text { Using the Respiratory gating technique in PET imaging to reduce activity smearing } \\
\text { and to improve accuracy in identifying the tumor. }\end{array}$ \\
\hline Fredberg Persson, Nygaard et al. 2011 & $\begin{array}{l}\text { Comparison between 3 CT methods of three-dimensional CT (3D-CT), four- } \\
\text { dimensional CT (4D-CT) and breath-hold CT (BH-CT) scan for evaluating the } \\
\text { tumor size. Results: breath-holding causes size of tumor to be presented smaller } \\
\text { in CT methods. }\end{array}$ \\
\hline He, O'Keefe et al. 2010 & $\begin{array}{l}\text { Proposed a new method by simulating PET acquisition and respiratory motion to } \\
\text { improve the image degradation caused by respiratory motion. Drawback: being } \\
\text { insensitive to non-axial motion }\end{array}$ \\
\hline Bettinardi, Picchio et al. 2010 & $\begin{array}{l}\text { Two solutions for the problem of respiratory gating to completely correct the } \\
\text { breathing motion: } \\
\text { 1) Increasing the PET acquisition time to obtain enough counting statistics. } \\
\text { 2) Take all counting statistics and integrate motion information before, during or } \\
\text { after the reconstruction process. }\end{array}$ \\
\hline Bépin, Daouk et al. 2014 & $\begin{array}{l}\text { Proposed a method to correct the motion in respiratory gated PET images and to } \\
\text { yield an appropriate distribution of the radioactivity concentration. Drawback: using } \\
\text { a very large time-varying system matrix for handling the respiratory motion and its } \\
\text { calculating is a major challenge for computational resources. }\end{array}$ \\
\hline $\begin{array}{l}\text { Aim: to define the role of respiratory gated 4D-PET/CT techniques to compensate } \\
\text { for degradation effects produced by respiratory motion on PET/CT images. }\end{array}$ \\
\hline
\end{tabular}




\section{CONCLUSIONS}

PET imaging provides quantitative functional information on diseases but motion caused by organ movement makes images blurred. Therefore, motion correction is of great importance for improving the quantity and quality of the obtained images. In this paper, we presented the state-of-the-art motion correction methods of image reconstruction that are commonly used for PET/CT imaging, as well as the recent advances in techniques applicable to PET and PET/CT images. In this review we described different motion correction methods, listed and compared which provides researchers and clinicians with detailed information and details that are well suited for any particular application.

\section{REFERENCE}

1. Ambrosini, V. et al. PET/CT imaging in different types of lung cancer: an overview. - European Journal of Radiology, 81, 2012, 5, 988-1001.

2. Ambwani, S. et al. Joint cardiac and respiratory motion correction and super-resolution reconstruction in coronary PET/CT. Biomedical Imaging: From Nano to Macro, IEEE International Symposium on 2011, 2011, IEEE.

3. Anishchenko, S. et al. Quality Estimation Of Motion Correction For Pet Brain Images. - Southern Federal University, 2012.

4. Bagci, U. et al. Joint segmentation of anatomical and functional images: Applications in quantification of lesions from PET, PET-CT, MRI-PET, and MRI-PET-CT images. - Medical Image Analysis, 2013, 8, 929-945.

5. Bai, W. and M. Brady. Motion correction and attenuation correction for respiratory gated PET images. - Medical Imaging, 30, IEEE Transactions on 2011, 2, 351-365.

6. Bettinardi, V. et al. Detection and compensation of organ/lesion motion using 4D-PET/CT respiratory gated acquisition techniques. - Radiotherapy and Oncology, 96, 2010, 3, 311-316.

7. Chang, G. et al. Joint correction of respiratory motion artifact and partial volume effect in lung/thoracic PET/CT imaging. - Medical Physics, 37, 2010, 12, 6221-6232.

8. Fayad, $\mathrm{H}$. et al. A generic respiratory motion model for motion correction in PET/CT. Nuclear Science Symposium Conference Record (NSS/MIC), 2010, IEEE 2010.

9. Ferlay, J. et al. GLOBOCAN 2012 v1. 0, Cancer Incidence and Mortality Worldwide: IARC CancerBase № 11. Lyon, France: International Agency for Research on Cancer; 2013. - Last accessed April 2014.

10. Fredberg Persson, G. et al. Artifacts in conventional computed tomography (CT) and free breathing four-dimensional CT induce uncertainty in gross tumor volume determination. - International Journal of Radiation Oncology* Biology* Physics, 80, 2011, 5, 1573-1580.

11. He, J., et al. Motion compensation based on dynamic data in PET acquisition. Biomedical Engineering and Informatics (BMEI), 2010 3rd International Conference on 2010, IEEE.

12. Keller, S. H. et al. Methods for motion correction evaluation using 18F-FDG human brain scans on a high-resolution PET scanner. - Journal of Nuclear Medicine 53, 2012, 3, 495-504. 
13. Lee, P. et al. Current concepts in F18 FDG PET/CT-based radiation therapy planning for lung cancer. - Frontiers in Oncology, 2, 2012.

14. Li, G. et al. Correction of motion-induced misalignment in co-registered PET/CT and MRI (T1/T2/ FLAIR) head images for stereotactic radiosurgery. - Journal of Applied Clinical Medical Physics, 12, 2010, 1.

15. Marache-Francisco, S. et al. Impact of respiratory motion correction on the detection of small lesions in whole-body PET imaging: a simulation study. Nuclear Science Symposium Conference Record (NSS/MIC), IEEE, 2010.

16. McClelland, J. R. et al. Respiratory motion models: A review. - Medical Image Analysis, 17, 2013, 1, $19-42$.

17. McQuaid, S. J. et al. A novel method for incorporating respiratory-matched attenuation correction in the motion correction of cardiac PET-CT studies. - Physics in Medicine and Biology, 56, 2011, 10, 2903.

18. Nehmeh, S. A. et al. Effect of respiratory gating on quantifying PET images of lung cancer. - Journal of Nuclear Medicine, 43, 2002, 7, 876-881.

19. Noonan, P. et al. Accurate markerless respiratory tracking for gated whole body PET using the Microsoft Kinect. Nuclear Science Symposium and Medical Imaging Conference (NSS/MIC), IEEE, 2012.

20. Pépin, A. et al. Management of respiratory motion in PET/computed tomography: the state of the art. - Nuclear Medicine Communications, 35, 2014, 2, 113.

21. Rahmim, A. et al. Strategies for motion tracking and correction in PET. - PET Clinics, 2, 2007, 2, 251-266.

22. Saha, G. B. Data Acquisition and Corrections. - Basics of PET Imaging, Springer, 2010: 41-69.

23. Takeuchi, S. et al. Impact of initial PET/CT staging in terms of clinical stage, management plan, and prognosis in 592 patients with non-small-cell lung cancer. - European Journal of Nuclear Medicine and Molecular Imaging, 41, 2014, 5, 906-914.

\section{Corresponding author:}

F. Nayyeri

Department of Computer Science

Faculty of Information Science and Technology

University Kebangsaan Malaysia

43600 UKM Bangi, Selangor, Malaysia

tel.: (+60)14 7015371

e-mail: fereshteh.nayyeri@gmail.com 